\title{
Potential Use of Ultrasound Speckle Tracking for Motion Management During Radiotherapy Preliminary Report
}

\author{
Jonathan M. Rubin, MD, PhD, Mary Feng, MD, Scott W. Hadley, PhD, J. Brian Fowlkes, PhD, \\ James D. Hamilton, PhD
}

Received August 16, 2011, from the Departments of Radiology (J.M.R., J.B.F.) and Radiation Oncology (M.F., S.W.H.), University of Michigan, Ann Arbor, Michigan USA; and Epsilon Imaging, Ann Arbor, Michigan USA (J.D.H.). Revision requested September 17, 2011. Revised manuscript accepted for publication September 30, 2011.

Dr Hamilton is an employee and shareholder of Epsilon Imaging.

Address correspondence to Jonathan $M$. Rubin, MD, PhD, University of Michigan Hospital, 1500 E Medical Center Dr, Ann Arbor, MI 48109 USA.

E-mail address:jrubin@umich.edu

\section{Abbreviations}

4DCT, 4-dimensional computed tomography; 1D, 1-dimensional; $R F$, radiofrequency; $R M S$, root mean square; ROI, region of interest; 3D, 3-dimensional; $2 D$, 2-dimensional
We prospectively evaluated real-time ultrasound speckle tracking for monitoring soft tissue motion for image-guided radiotherapy. Two human volunteers and 1 patient with a proven hepatocellular carcinoma, who was being prepared for radiation therapy treatment, were scanned using a clinical ultrasound scanner modified to acquire and store radiofrequency signals. Scans were performed of the liver in the volunteers and the patient. In the patient, the speckle-tracking results were compared to those measured on a treatment-planning 4-dimensional computed tomogram with tumors contoured manually in each phase and with estimates made by hand on gray scale ultrasound images. The surface of the right lung and the prostate were scanned in a volunteer. The liver and lung surface were scanned during respiration. To simulate prostate motion, the ultrasound probe was rocked in an anterior-posterior direction. The correlation coefficients of all motion measurements were significantly correlated at all sites $(P<.00001$ for all sites) with 0 time delays. Ultrasound speckle-tracking motion estimates of tumor motion were within $2 \mathrm{~mm}$ of estimates made by hand tracking on gray scale ultrasound images and the 4-dimensional computed tomogram. The total tumor motion was greater than $20 \mathrm{~mm}$. The angular displacement of the prostate was within 0.02 radians $\left(1.1^{\circ}\right)$ with displacements measured by hand. Speckle tracking could be used to monitor organ motion during radiotherapy.

Key Words — radiation oncology; tissue motion; ultrasound; ultrasound speckle; ultrasound speckle tracking

$\Lambda$ s the conformality of radiotherapy improves, the importance of accounting for geometric uncertainties in tumor and normal tissue positions increases. In mobile regions such as the thorax and abdomen, tumor motion has been described as up to 2 to $3 \mathrm{~cm}$ for lung, pancreas, and liver malignancies, mostly in the superior-inferior direction, although anterior-posterior motion can also be substantial. ${ }^{1-3}$ Even in the pelvis, prostate motion can be considerable. ${ }^{4}$

Traditionally, large uniform margins have been added to the intended treatment target to generate a planning target volume, with the intent of covering the full range of motion with full-dose radiation. This process results in large radiotherapy fields, poor tolerance of treatment, and limited radiation doses if organs at risk are nearby. 
As tumor motion has been better characterized, it is now apparent that motion varies from patient to patient, day to day, and even breath to breath. Even a "large" 2 -cm margin may be inadequate in certain situations while extreme in others.

To both ensure target coverage and minimize radiation dose to organs at risk, motion must be accounted for in both the planning and delivery of radiotherapy. Respiratory-correlated 4-dimensional computed tomography (4DCT) can capture the motion of internal anatomy. ${ }^{5,6}$ However, this method is performed before the procedure and may not reflect the patient's respiratory motion during treatment. Respiratory signals during therapy can be measured from external marker tracking of abdominal motion or respiratory belts. ${ }^{7}$ However, external signals can have a poor correlation with the actual motion of internal anatomy. ${ }^{1,6,8-10}$

Thus, many groups use internal gold or carbon fiducials, or electromagnetic tracking devices implanted within or near tumors, to monitor the target position. ${ }^{11-13}$ Although these fiducials can provide important information, they require an invasive procedure for implantation.

A conceptually simple way to manage motion is to suspend it through the use of a device such as active breathing control, which allows radiation oncologists to minimize the planning target volume to as little as $5 \mathrm{~mm}$ by eliminating the respiratory motion component of geometric uncertainty. ${ }^{11}$ Active breathing control ensures target coverage and minimizes unnecessary radiation to organs at risk. Although this method has allowed for dose escalation with reduced side effects, it is uncomfortable for patients and time and labor intensive in the clinic.

Ultrasound imaging is a robust and proven method for evaluating tissue motion in real time. ${ }^{14-22}$ Ultrasound is particularly attractive in this regard because it contains speckle, which provides robust, densely positioned targets for tracking tissue motion. ${ }^{23}$ Speckle tracking of tissue motion has proven to be highly accurate, easily to submillimeter accuracies at standard ultrasonic frequencies, and has led to a large and ever-expanding number of medical applications that involve ultrasound speckle tracking for motion estimates. These include elasticity imaging, blood flow measurements, cardiac strain imaging, extended field 2-dimensional (2D) imaging, and 3-dimensional (3D) image production from $2 \mathrm{D}$ slices. ${ }^{15,17-22,24,25}$

A series of papers have suggested that ultrasound speckle tracking could be used to monitor physiologic motion during radiation oncology treatments. ${ }^{26-29}$ They displayed the feasibility of ultrasound speckle tracking or feature/lesion tracking in a radiation treatment environ- ment. Tracking has been tested in a speckle-containing phantom while a treatment beam was on. ${ }^{27}$ Furthermore, Harris et $\mathrm{al}^{28}$ also showed that speckle tracking could be used to monitor motion of the liver in normal human volunteers in $3 \mathrm{D}$ during respirations, and that the motion estimated using speckle tracking compared favorably to the motion predicted when an operator interactively tracked features in the liver. Their tracking was performed offline.

In this study, we performed real-time 2D tracking of liver respiratory motion using specially designed software originally designed for tracking cardiac motion. We demonstrate accurate tracking of liver motion, and furthermore, we demonstrate the ability to track the lung surface and simulated prostate motion in real time. Finally, we compared the estimated respiratory-induced motion using speckle tracking of a liver containing a hepatocellular carcinoma with motion estimates made by human operators tracking the tumor's motion on ultrasound images and on a 4DCT scan performed before treatment.

\section{Materials and Methods}

Two healthy volunteers and a 78-year-old man with a 4.2$\mathrm{cm}$ hepatocellular carcinoma in the right lobe of the liver were enrolled in a University of Michigan Institutional Review Board-approved prospective study with written informed consent obtained in all cases. The ultrasound scans were performed using a clinical ultrasound scanner (Epsilon Imaging; Ann Arbor, MI) specially designed to acquire ultrasonic radiofrequency $(\mathrm{RF})$ signals at very high acquisition rates of more than 200 frames per second. These high frame rates are crucial to the primary application for the device, which is measurement of cardiac strains during heart motion, and this device has proven to be highly accurate at measuring tissue motion at these frame rates. ${ }^{30}$ This high frame rate also made it possible for us to modulate the tracking rate for the different applications. Strain and strain rate images describing tissue deformation and tissue velocity images describing tissue motion can be displayed at real-time rates on the order of 30 frames per second. Furthermore, motion data can be analyzed offline using a workstation-based software application (EchoInsight; Epsilon Imaging).

Tracking was performed on the RF signals. The tracking method is based on complex (ie, real and imaginary numbers) cross-correlation algorithms as previously described..$^{17}$ Briefly, this phase-sensitive method tracks speckle motion by comparing RF signatures from acquisition to acquisition within small regions of interest (ROIs) and then assigns displacements to the regions 
based on maximum correlation coefficients. The location with the highest correlation coefficient match is the assigned position for each displacement. The method is well accepted ${ }^{17}$ and can very accurately estimate tissue motion to submillimeter accuracies along the ultrasound beam at standard diagnostic ultrasound carrier frequencies.

\section{Scanning Technique}

For the liver images and the transperineal images of the prostate, we used an approximately $2.5-\mathrm{MHz}$ phased array probe. The RF sampling rate during these acquisitions was $10 \mathrm{MHz}$ at a frame rate of $63 \mathrm{~Hz}$.

Liver scanning was performed between the ribs, and images were generated in a cephalad-caudad direction so that motion across the image would correspond to standard inspiration and expiration displacements. The method does not require that specific hepatic structures be identified but rather that only liver parenchyma be positioned within the scan (Figure 1).

For evaluation of the prostate, transperineal images were produced in the longitudinal orientation as well (Figure 2). Since prostate displacements are produced by random and sometimes infrequent contractions of adjacent bowel or bladder filling, we could not reliably produce this motion in an experimental setting. To simulate prostate motion, we rocked the ultrasound scan head in an anterior-posterior direction. The scan head motion will produce the appearance of motion in the stationary prostate gland, and the apparent displacements will appear as motion across the image. The speckle displacements will be similar to what would occur with an actually moving prostate and a stationary ultrasound scan head. We rocked the scan head sufficiently to produce a simulated total displacement of the prostate of about $1 \mathrm{~cm}$ on average, which covers the typical intrafraction displacements of the prostate during radiation therapy treatments. ${ }^{35,36}$ Multiple experiments performed by us and others have shown that displacement estimates made by speckle-tracking RF signals in ultrasound phantoms and in vivo are highly accurate. ${ }^{27,28,30}$ Since the motion was angular, the linear displacement varied across the scan image with displacements increasing with depth from the scan head. We also rocked the ultrasound probe sufficiently rapidly to simulate displacement rates of about 2 to $3 \mathrm{~cm} / \mathrm{s}$ depending on the distance from the ultrasound probe.

Scans of the right lung surface were made using an approximately 6.0-MHz linear array transducer (Figure 3). The scans were performed intercostally, so the absolute orientation was defined by the angle of each interspace rel- ative to the long axis of the body. Thus, the motion was not perfectly in the cephalad-caudad direction, although we tried to scan as close to the cephalad-caudad direction as possible. Since ultrasound does not propagate beyond airsoft tissue boundaries, the lung surface appears as a very bright line across each ultrasound image (Figure 3 ). The RF sampling rate for the lung surface acquisitions was 20 $\mathrm{MHz}$ at a frame rate of $105 \mathrm{~Hz}$.

The 4DCT scans were performed with a Brilliance Big Bore 16-slice CT scanner (Philips Healthcare, Cleveland, $\mathrm{OH})$. The scanning parameters were $120 \mathrm{kVp}, 600 \mathrm{mAs}$, 3$\mathrm{mm}$ slice thickness, 10 equally spaced breathing phases, 0.08 pitch, $3.8-\mathrm{mm} / \mathrm{s}$ couch velocity, and 0.5 -second tube rotation. A respiratory trace was obtained using a bellows and was correlated with CT images. The respiratory cycle was divided into 10 equally spaced phases, from maximum inhale to maximum exhale, and the $\mathrm{CT}$ images were sorted into the appropriate phases.

\section{Motion Capturing}

For each organ in the healthy volunteers, 3 ROIs were selected. The selected ROIs in the liver, prostate, and lung surface had dimensions of approximately $1.0 \times 1.5 \mathrm{~cm}$, $1 \mathrm{~cm}^{2}$, and $2 \times 5 \mathrm{~mm}$, respectively. Each of the motion sequences were captured over 2 -second intervals. At least 1.6 seconds of each interval was used for tracking. The ROIs moved in $2 \mathrm{D}$ with the moving speckle. For purposes of display, the 1 dimensional (1D) component along the primary motion direction (ie, the direction with the greatest motion) was plotted with respect to time on one plot, and we also present plots of the corresponding orthogonal motion in a second plot (Figures 1B and 3F). The plots represent the motion of the center of each ROI. For the liver, the primary axis of motion tracking was nearly cephaladcaudad, while the lung surface motion direction was somewhat oblique as mentioned above. Within any respiratory cycle, each different ROI in an image was represented by a different color (Figures 1-3).

In the patient with hepatocellular carcinoma, the motion of the tumor was tracked in a coronal plane in a cephalad-caudad direction similar to the healthy volunteers. The tracking was performed from full inspiration to full expiration over 2 seconds on the ultrasound scans. An ROI similar in size to those used in the volunteers was drawn around the tumor. The speckle in the ROI was again tracked over the 2-second interval. Then the images from the sequence were transferred to a personal computer, and the motion of the tumor was tracked by hand on the computer display by one of the investigators (J.M.R.). The tumor motion was tracked 4 times. 
These values were compared to the displacement estimate on the 4DCT. The liver tumor was contoured on the inhale and exhale positions by a radiation oncologist (M.F.) (Figure 4). The displacement of the centroid of the tumor between the two phases was calculated.

Figure 1. A, Longitudinal scan of the right lobe of the liver used for tracking. There are 3 rectangular regions of interest (ROls), indicated by red, green, and blue ovals, marked in the liver. Each specifically tracked region is the rectangle within each oval. These were tracked over a 2-second respiratory cycle. The right kidney (thin arrows) is noted deep to the liver, and the diaphragm (large arrow) is noted along the left side of the image. The estimated linear displacement measured on the ultrasound scanner screen for comparisons with the speckle tracking was made at the red ellipse (see B and C). B, Composite showing 3 images representing different locations in the liver of a healthy volunteer taken during the 2-second breath corresponding to $\mathbf{A}$. Three points labeled with red, blue, and green circles correspond to those in $\mathbf{A}$. Below the 3 images are the time profiles of the motions of the 3 positions during the respiration. The profiles, which are labeled with the colors of their corresponding points, represent the average motion of the speckle in the 3 different ROls. The profiles in the plot labeled $x$ motion correspond to the average motion in the cephalad-caudad direction, while the profiles in the plot labeled y motion correspond to the orthogonal motion to those shown in x motion image. Relatively little motion is seen in the orthogonal direction. Notice that the x motions are highly correlated, although there is some variation. Some of this variation could actually be due to deformation in the liver during respiration. ${ }^{31-34} \mathbf{C}$ (opposite page), This image shows the change in position for the tracking measurement made by hand by a human observer from $\mathbf{A}$ to $\mathbf{B}$, which is full expiration to full inspiration on the left side of the image, and then $\mathbf{B}$ to $\mathbf{C}$, which is full inspiration to full expiration on the right side. The displacements are represented by the yellow lines in each image, with the liverkidney boundary marked by a white arrow in each segment. (This motion is represented by the red curve in the displacement-time plot in the middle of the image.) Notice that $A-B$ does not equal $B-C$, which shows how respiratory displacements can vary even in relatively controlled circumstances such as an imaging experiment such as this. The corresponding positions in the respiratory cycle for each image are depicted by a blue arrow pointing from each image frame to the corresponding time point in the plot. The A-to-B displacement is $16 \mathrm{~mm}$; the B-to-C displacement is $21.5 \mathrm{~mm}$.

A

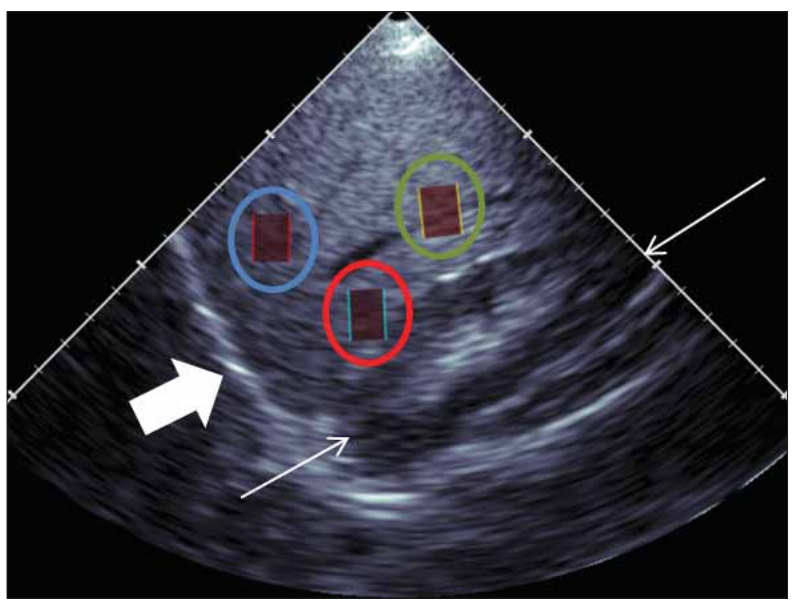

For the prostate, the gland was imaged transperoneally in the sagittal orientation. The motion was artificially induced by rocking the ultrasound scan head so the gland appeared to move in an approximately anterior-posterior direction. The true motion was angular with the apex of the angle positioned at the peroneal surface at the site of contact of the scan head. For the lung surface, the ROIs were placed along the lung surface-soft tissue boundary. Regions of interest placed beyond the boundary would only be registering noise. During respiration, the boundary moved, and each ROI moved with the moving boundary.

Radiofrequency data from the lung surface were filtered to remove the strong static specular reflectors and static noise signals at the air-soft tissue interface. A highpass, finite impulse response, 3-point Hanning high-pass filter with coefficients $(-0.25,0.5$, and -0.25$)$ was applied between successive RF frames (Figure 3D). Similar filters, often called wall or clutter filters, are typically used in ultrasound Doppler processing to remove stationary tissue signals during blood flow measurements and were useful in removing the strong static lung surface echoes in this application.

B
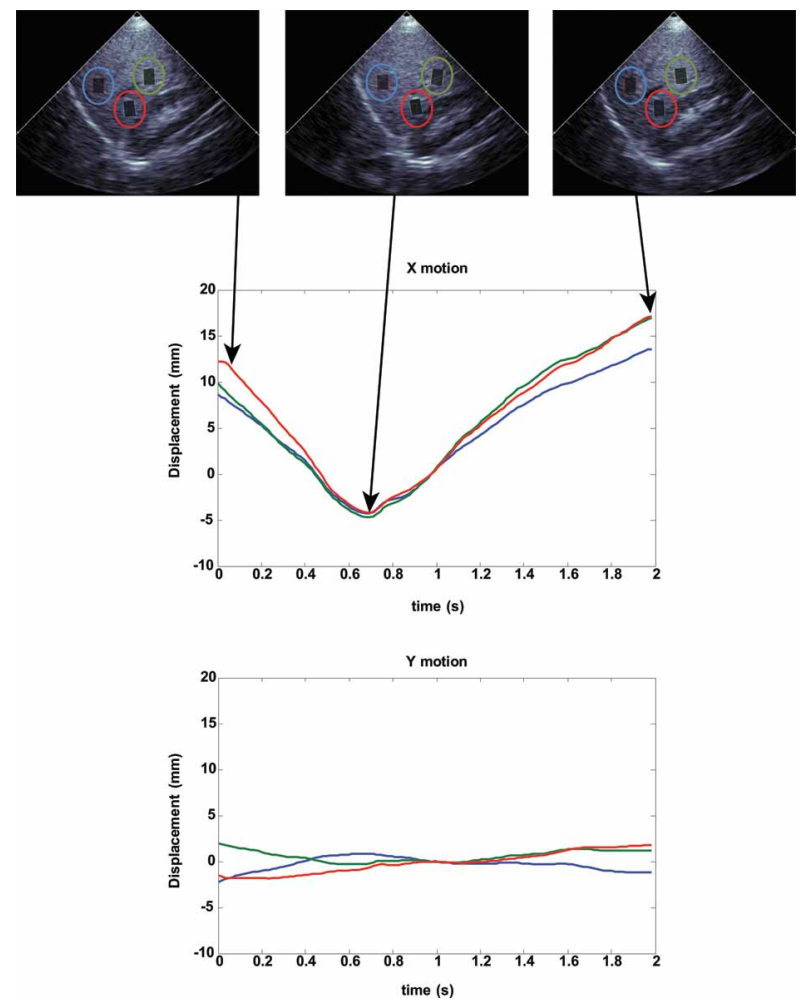
C

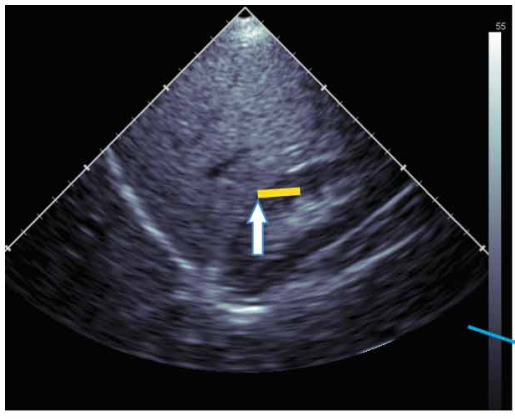

A to B motion
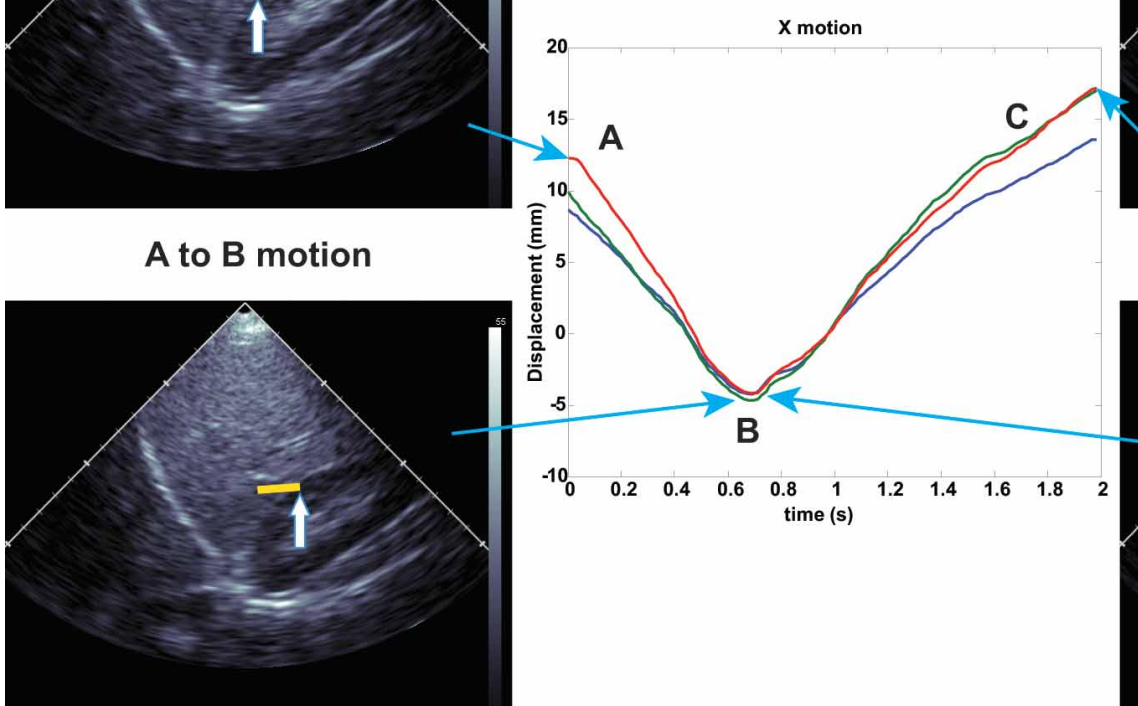

time (s)

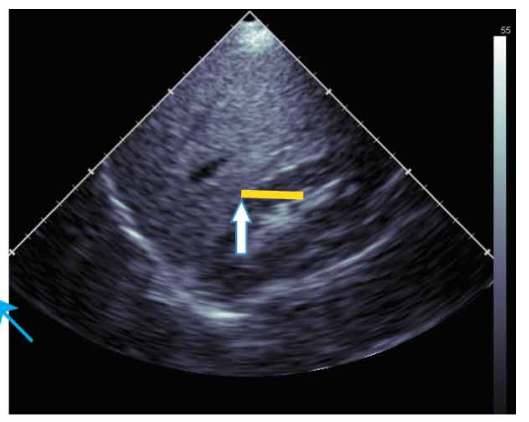

B to $\mathrm{C}$ motion

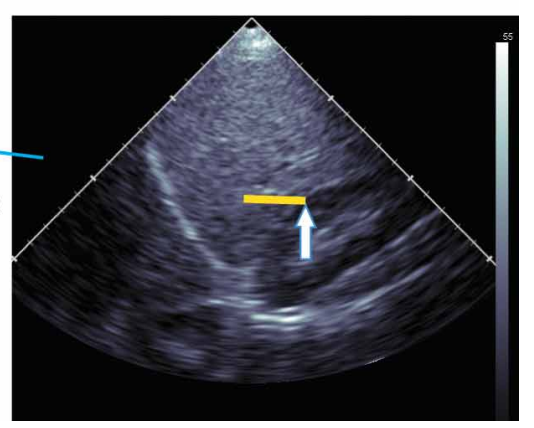

Figure 2. A, Two-dimensional ultrasound scan of the prostate. There are 3 approximately $1-\mathrm{cm}$ square regions of interest (ROIs) in the gland marked with green, red, and blue ovals. The cephalad margins of the gland are marked with white arrows. Thescan apex is on the perineum. The estimated linear displacement measured on the ultrasound scanner screen for comparisons with the speckle tracking was made at the red ellipse. Anterior, posterior, and cephalad directions are marked on the image. B, Angular tracking profiles for the $3 \mathrm{ROIs}$ in the prostate shown in $\mathbf{A}$. The profiles in the plot correspond to the motion of the center point in each ROI. The 2 images at the top of the composite show the displacements of the angular sweep at a depth of $4 \mathrm{~cm}$ from the transducer where the absolute linear displacement distance is represented by the yellow line in each image. The white arrow in each image marks the position of a specific tracked point in each image. This displacement was tracked interactively on the cine loop of the moving prostate made by rocking the transducer. The linear displacement of the tracked point was $8.5 \mathrm{~mm}$ at $4 \mathrm{~cm}$ corresponding to 0.21 radians $\left(12^{\circ}\right)$. A

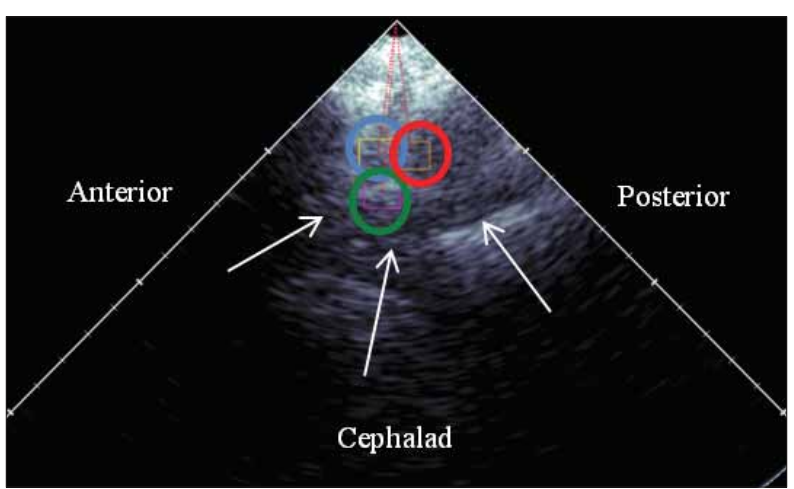

B
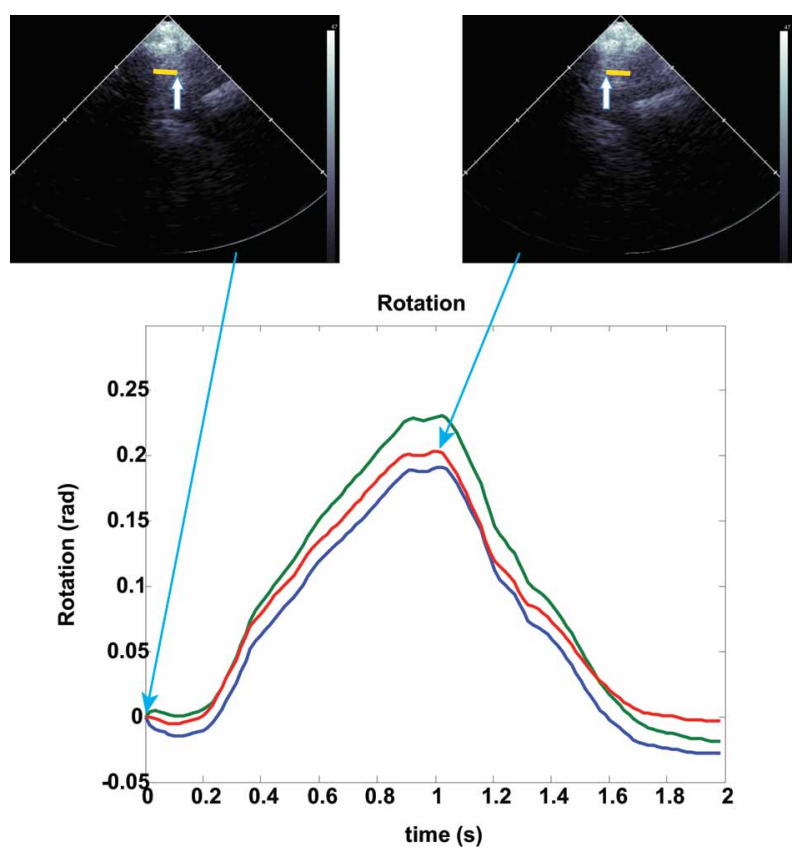
Figure 3. A, Unfiltered gray scale image of the surface of the right lung in a near sagittal orientation. The lung surface is marked with arrows. The static background echoes are visible in this representation. B, Filtered image of the surface of the lung. The surface of the lung is again marked with arrows. The static soft tissue echoes superficial to the lung surface have been removed by the filter. Only the lung surface and reverberation noise deep to the surface are now visible. This image has higher contrast than the unfiltered image. $\mathbf{C}$, Filtered image of the surface of the lung now with three $2 \times 5$-mm rectangular regions of interest (ROIs) lying along the lung-soft tissue boundary (arrows). Each $\mathrm{ROI}$ is surrounded by an oval: blue, red, or green. The B-mode image is formed from high-pass-filtered (ie, clutter-filtered) radiofrequency data (B). Stationary signals arising from specular reflections and noise at the lung-tissue interface and the stationary surrounding tissue have been reduced or eliminated. D (opposite page), Frequency response of the high-pass finite impulse response filter used to remove the static signals at the lung-soft tissue boundary. $\mathbf{E}$, Motion of the 3 ROls marked in $\mathbf{C}$ over a portion of the respiratory cycle. Notice that the final 1 second from 0.6 to 1.6 seconds corresponds to full inspiration to full expiration. The profiles in the plot represent the motion of the center point in each ROI. Again, they are highly correlated with some tracking variation. The total displacement is about $1 \mathrm{~cm}$. F, Simultaneous motion of the 3 ROls marked in $\mathbf{E}$ over the same respiratory cycle but in the orthogonal direction to the motion shown in $\mathbf{E}$. The profiles in the plot correspond to the motion of the center point in each ROI. Note that there is very little motion in this direction compared to $\mathbf{E}$. G. Displacement of the lung boundary based on tracking the unfiltered image in $\mathbf{A}$. Notice that the magnitude of the measured displacement is 3 to $4 \mathrm{~mm}$ instead of the $1 \mathrm{~cm}$ tracked in $\mathbf{E}$. The decreased measured motion is due to the predominance of stationary echoes from either clutter or stationary specular reflectors in the image, which dominate the correlation tracking.

A

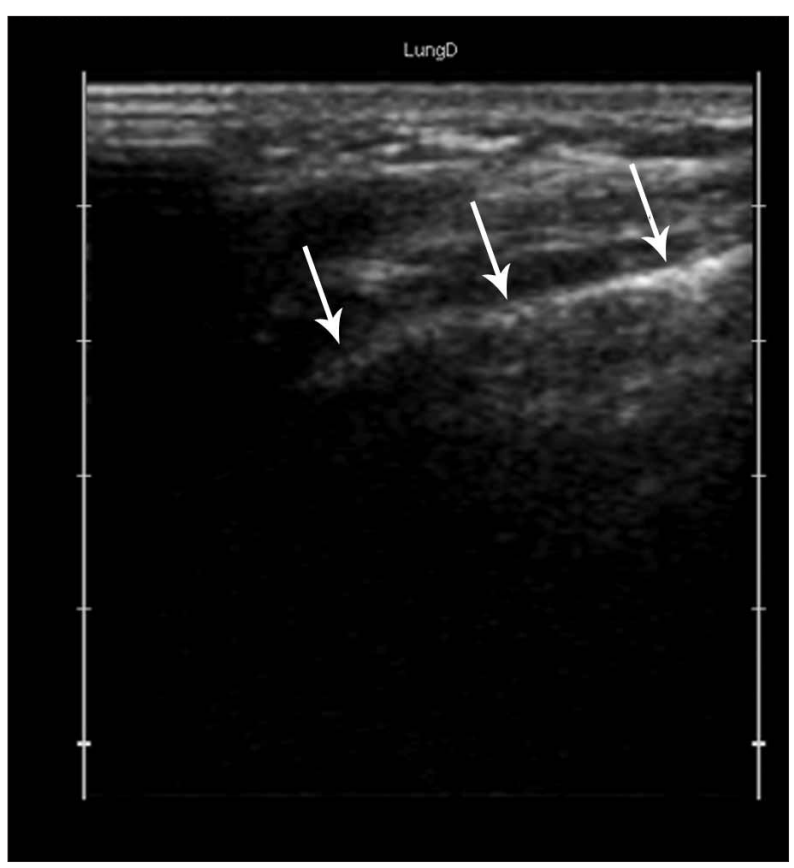

B

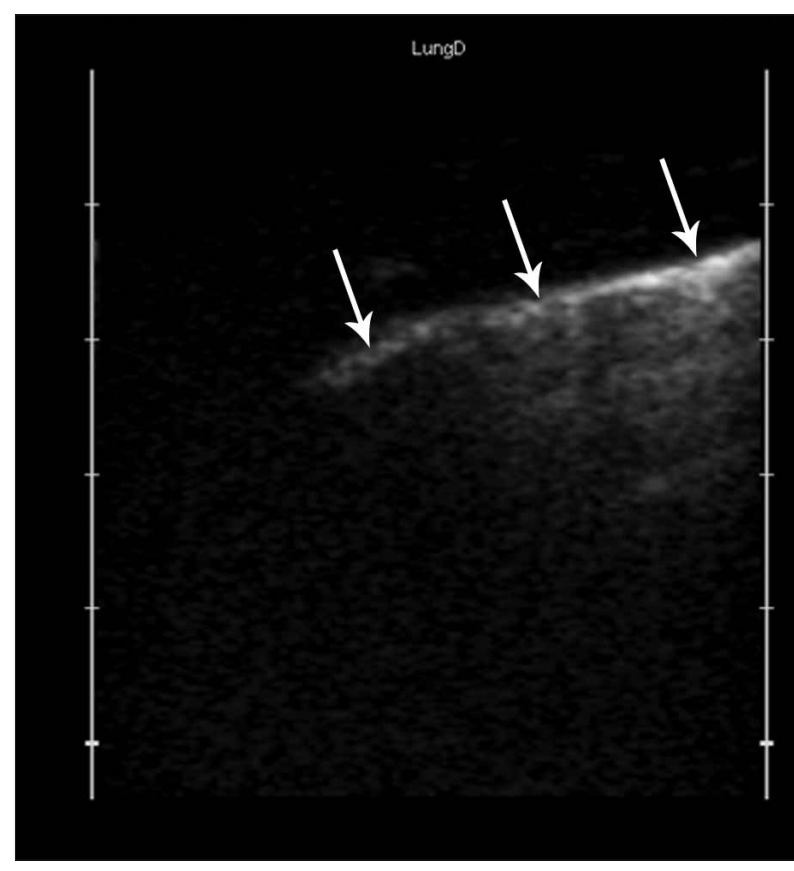

C

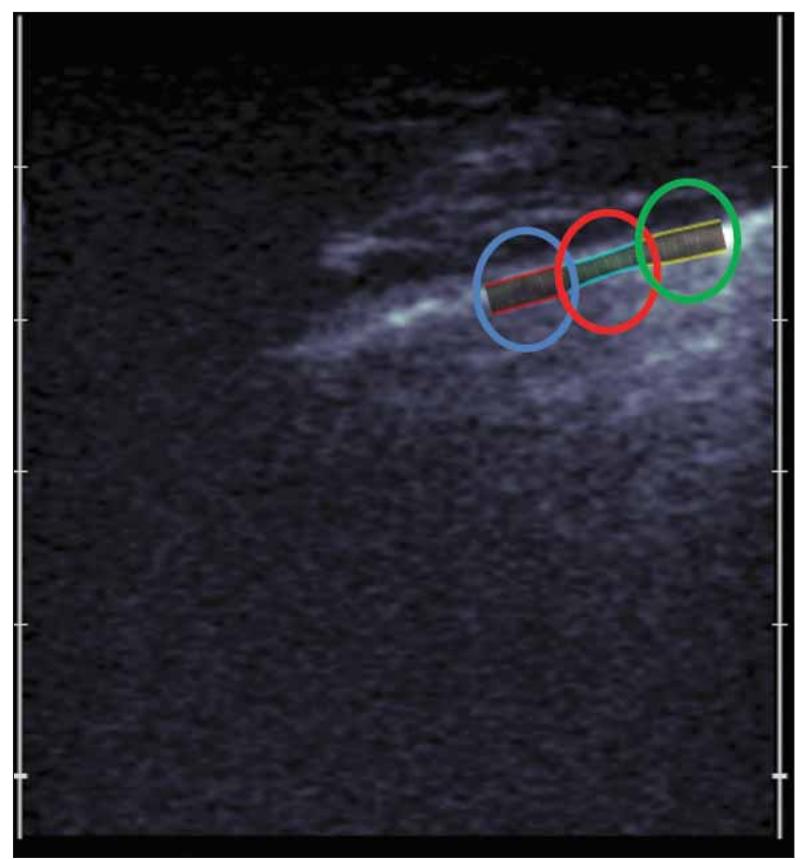


D

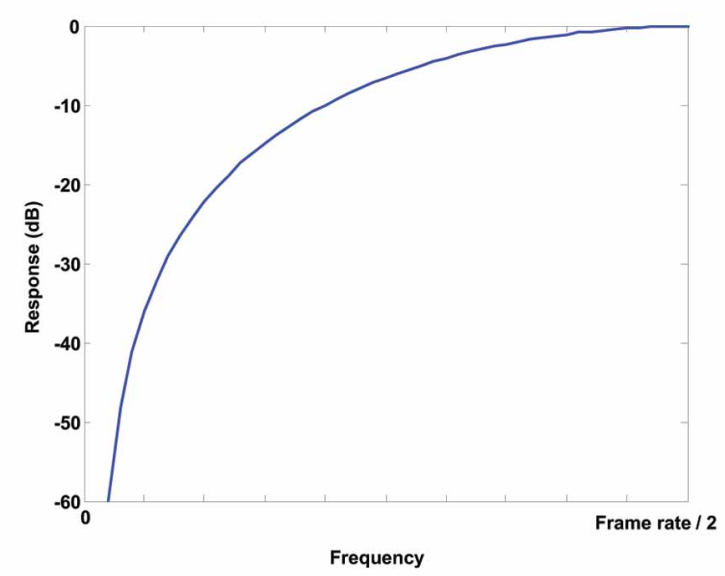

E

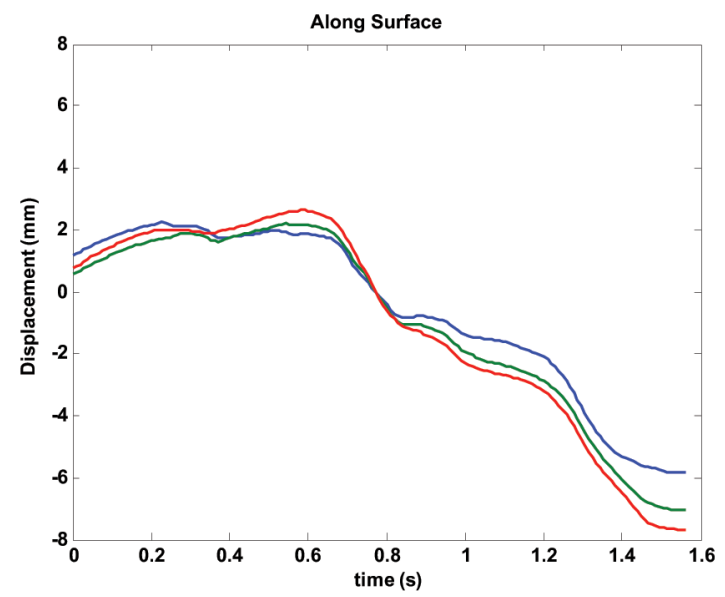

$\mathbf{F}$

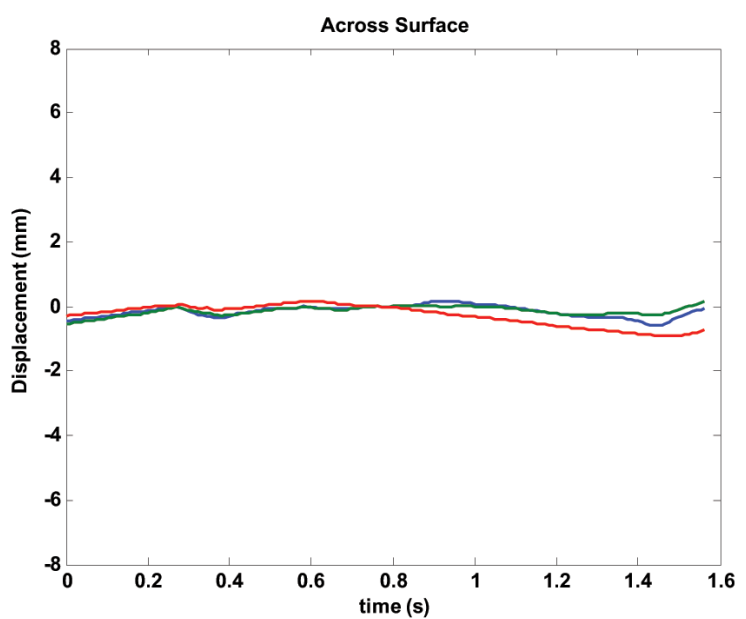

\section{Statistical Analysis}

We tracked the absolute motion of each ROI, and these motions were displayed graphically as $1 \mathrm{D}$ displacements as a function of time. We then performed 2-time temporal correlations among all of the ROIs in an acquisition to see how similar the tracking was throughout each motion acquisition. We compared the phase relationships between the different acquisitions to see if there were any systematic time delays among the acquisitions. Perfect tracking and identical motion would have temporal correlations of 1 and time/phase delays of 0 . We compared the pair correlations using a null hypothesis of 0 correlation between the pair of points. We chose a significance level of $P<.05$ as a significant correlation between points. Furthermore, we measured the total displacement of the points in the liver and lung surface over a standard breath and estimated the root mean square (RMS) variations in the total displacements over the respiratory cycle among 3 selected positions in the liver and lung surface.

For the prostate, we made similar measurements; however, since the motion was angular by design, points farther away from the point at which the transducer was being rocked would move faster and farther than those closer to the transducer. Therefore, we performed the correlations in angular coordinates and measured the correlations in angular displacements. The criterion for rejection of the null hypothesis, ie, no correlation in the angular motion, was still $P<.05$, and we estimated the tracking error between regions in RMS variation in the overall angular displacement through one sweep of the transducer from posterior to anterior and back to the starting point.

G

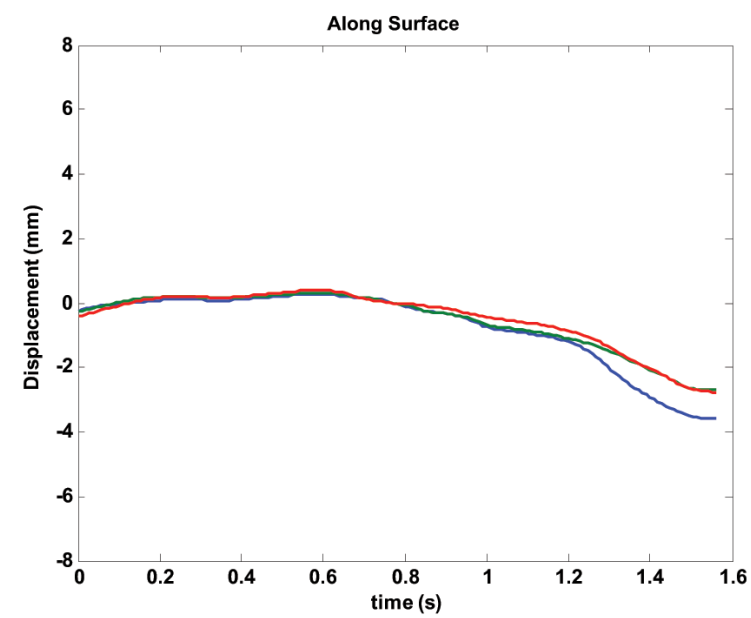


Finally, as a benchmark for the motion estimates in the liver and prostate, we measured the displacements of the organs using measurement calipers provided by the analysis software. Features were identified in the original image of each sequence, visually tracked by a human operator (J.M.R. or J.D.H.), and measured by hand to determine their overall displacement. In the liver, the speckle-tracking displacements were compared to the liver-right kidney boundary. Three measurements of the boundary motion were made. For the prostate, the boundary of the gland was marked and tracked at a depth of $4 \mathrm{~cm}$ from the contact point on the perineum. Using the atan to estimate the angular displacements, 3 estimates of the angular displacement were calculated. A similar comparison could not be made of lung surface motion because there were no identifiable anatomic structures that could be interactively tracked. We then compared the speckle-tracking total displacements to those made using standard caliper measurements. Since only a few measurements were made in each case, we used these measurements only to confirm that the speckle-tracking estimates are "close" to those estimates using visual features.

For the patient with hepatocellular carcinoma, the tumor displacement was estimated using the centroid of the tumor contoured on end-inhale and end-exhale CT images reconstructed from 4DCT. Again, since only 1 patient with liver cancer was studied in this preliminary work, a statistical comparison of the methods cannot be performed. However, we display the ultrasound-produced displacement estimates along with those from the 4DCT for comparison (Table 1).

\section{Results}

For the normal liver tracking, the average total peak inhalation-to-peak exhalation and vice versa displacements were measured at 3 sites. The RMS variations among the measurements, the average correlation coefficients between the measurements in the liver, the fractional tracking varia-

Table 1. Comparison of 4-Dimensional Computed Tomography, Tracking by Hand, and Speckle Tracking of a Liver Tumor

\begin{tabular}{lc}
\hline Measurement Method & Displacement, $\mathbf{m m}$ \\
\hline 4DCT & $21.2^{\mathrm{a}}$ \\
On-screen tracking by hand & $22.9 \pm 1.1$ \\
Speckle tracking & $20.7 \pm 0.3$ \\
\hline
\end{tabular}

Relative displacement estimates of a right hepatic lobe hepatocellular carcinoma during full expiration among 4-dimensional computed tomography (4DCT), on-screen motion tracking by a human volunteer, and automated speckle tracking. Values are mean \pm SD. aOne measurement. tion among the measurements $[(\mathrm{RMS}$ variation $) /($ average total peak-to-peak motion) ], and the approximate peak velocity in the primary motion direction for the liver are shown for speckle tracking. For comparison, measurements of the interactive tracking by a human of the liverright kidney boundary are shown (Table 2). Figure 1 depicts 3 selected regions in the liver whose motions were tracked using speckle tracking and direct measurement by a human observer from the acquired video. The same holds true for the lung surface, except we captured only the peak inhalation-to-exhalation portion of the cycle in its entirety (Table 2). Figure 3, A-F, shows the lung motion, filtered and unfiltered, as depicted by speckle tracking.

Table 3 and Figure 2 show the apparent angular displacement results induced in the prostate of a volunteer by rocking the transducer on the perineum. The angles calculated from the human interactive tracking results are the atan of the horizontal displacement of the margin of the prostate at a $4-\mathrm{cm}$ depth.

The liver images are from volunteer 1 , and the lung and prostate images are from volunteer 2 . In all cases, maximum temporal correlations always occurred with 0 phase shift between plots, suggesting no time delays among the points.

For the patient with the right lobe hepatocellular carcinoma, the comparative results among the 3 tracking methods are shown in Table 1 with the images showing the tumor motion on 4DCT (Figure 4A), human tracking using the gray scale video output (Figure 4B), and speckle tracking for motion estimation (Figure 4C). The ultrasound and CT displacements are all full inspiration to full expiration. The preliminary comparisons are very concordant.

\section{Discussion}

We were able to use $2 \mathrm{D}$ ultrasound speckle tracking to map the motions of the liver, right lung surface, and prostate (Figures 1-3) in healthy volunteers and a tumor in a patient with hepatocellular carcinoma (Figure 4). As demonstrated, the speckle tracking of tissue motion is highly reproducible, precise, and acquired in real time. Other important features are that no implantable markers and no additional radiation are necessary to acquire images. The tracking ROIs are generic and not restricted to specific portions of each organ's anatomy. Motion estimates are based on tracking the speckle inherent in the ultrasound images. Ultrasound speckle tracking is robust and extremely well characterized. ${ }^{17}$ Multiple previous works have demonstrated the accuracy of tissue displacements based on speckle tracking in vivo and in vitro..$^{27,28,37}$ These accuracies 
Table 2. Liver and Lung Motions

\begin{tabular}{lccccc}
\hline Organ & $\begin{array}{c}\text { Average Displacement } \\
\text { Using Speckle } \\
\text { Tracking } \pm \text { SD, } \mathbf{m m}\end{array}$ & $\begin{array}{c}\text { Average Correlation } \\
\text { Coefficient Between } \\
\text { Regions } \pm \text { SD }\end{array}$ & $\begin{array}{c}\text { Fractional } \\
\text { Linear Tracking } \\
\text { Variation, \% }\end{array}$ & $\begin{array}{c}\text { Peak Velocity } \\
\text { in Primary Motion } \\
\text { Direction, mm/s }\end{array}$ & $\begin{array}{c}\text { Freehand Distance } \\
\text { Estimate } \pm \text { SD, mm }\end{array}$ \\
\hline Liver (e to i) & $14.3 \pm 2.1$ & $0.9980 \pm 0.0012^{\mathrm{b}}$ & 14.7 & $\approx 30$ & $14.9 \pm 1.4$ \\
Liver (i to e) & $20.0 \pm 2.6$ & $0.9960 \pm 0.0016^{\mathrm{b}}$ & 13.0 & $\approx 30$ & $22.7 \pm 1.5$ \\
Lung surface & $10.0 \pm 0.72$ & $0.9987 \pm 0.0079^{\mathrm{b}}$ & 7.2 & $\approx 25$ & $\mathrm{NA}$ \\
\hline
\end{tabular}

Motion characteristics for the liver and lung surface. The liver motion was divided into full expiration (e) to full inspiration (i) and full inspiration to full expiration displacements separately. We were able to divide the liver motion because of the rapid respiration rate during the liver motion acquisition. aEstimate made from linear displacement of the red ellipse in Figure $1 \mathrm{~A}$. a $P<.00001$.

are on the order of millimeter and submillimeter levels. ${ }^{27,28,37}$ The degrees of accuracy can be beam profile and depth dependent, but these properties are well known and should be easily accountable when ultrasound is used to monitor tissue motion during radiation oncology treatments. ${ }^{38}$

We noted that fractional tracking variations were from about $7 \%$ to $15 \%$, with the liver being the highest. Although these numbers are very small for comparison, the fact that the liver has the highest variation is not really a surprise because it is well known that there is actually tissue deformation occurring in the liver when it is being displaced by respirations. ${ }^{31-34}$ This deformation would add variability to the liver displacement measures, even though this variability would be a reflection of the true liver motion. The angular variations in our prostate scans are solid-body motions, which would have no such variations, while the lung motion is a complex volumetric motion, ${ }^{39}$ and we are only imaging the surface. Thus, the lung might be deforming in ways we cannot detect with our technique. A larger study would be necessary to analyze these effects.

Speckle tracking would be very simple to implement for daily radiation treatments. All one need do is attach an ultrasound transducer to the patient's body and produce an image somewhere in the organ of interest. The trans- ducer itself could actually represent nothing more than an attachable ultrasound transducer. It need, in theory, be no larger than a standard electrocardiographic lead. In fact, multiple elements could be attached similar to multiple electrocardiographic leads, with each element acting as a reliability check for the other elements. Furthermore, with multiple elements, it will be possible to estimate actual deformations in motion or to estimate 3D motion using simple $1 \mathrm{D}$ transducer arrays.

Another big advantage of the method is that the quality of the actual images can be quite low. Since we are only interested in identifying the target, speckle-containing organ, specific structural details such as portal veins, bile ducts, and hepatic veins in the liver or the peripheral zone, urethra, and seminal vesicles for prostate scanning need not be seen. Therefore, one can use low-frequency, smallaperture, phased array probes that guarantee penetration at the expense of spatial resolution. The spatial resolution trade-off would be on the order of $1 \mathrm{~mm}$ or less, so the practical cost in terms of tracking would be low.

Of course, ultrasound imaging only gives motion relative to the ultrasound probe. For purposes of full positioning, it will be important to know where the probes are relative to the room coordinate system, so if the patient moves his or her body, the radiation therapy beam can be

Table 3. Prostate Motion

\begin{tabular}{|c|c|c|c|c|c|}
\hline Organ & $\begin{array}{l}\text { Average Angular } \\
\text { Rotation } \\
\text { Using Speckle } \\
\text { Tracking, } \\
\text { radians }\end{array}$ & $\begin{array}{l}\text { Average } \\
\text { Correlation } \\
\text { Coefficient } \\
\text { Between } \\
\text { Regions }\end{array}$ & $\begin{array}{c}\text { Fractional } \\
\text { Angular } \\
\text { Tracking } \\
\text { Error, \% }\end{array}$ & $\begin{array}{l}\text { Peak Velocity } \\
\text { Across } \\
\text { Beam at the } \\
\text { Position of the } \\
\text { Prostate, } \mathrm{mm} / \mathrm{s}\end{array}$ & $\begin{array}{l}\text { Freehand } \\
\text { Rotational } \\
\text { Measurement } \\
\text { Estimate, } \\
\text { radians }^{\mathrm{a}}\end{array}$ \\
\hline Prostate & $0.23 \pm 0.02$ & $0.9980 \pm 0.0014^{b}$ & 8.7 & $\approx 25$ & $0.25 \pm 0.01$ \\
\hline
\end{tabular}

Detected artificial motion produced in the prostate by rocking the ultrasound probe. The peak velocity across the beam was estimated at about $5 \mathrm{~cm}$ from the transducer. The velocity will, of course, be depth dependent. Values are \pm SD where applicable.

aEstimated angular displacement measured on the ultrasound scanner screen for comparsion with speckle tracking was made at $4 \mathrm{~cm}$ using atan angle approximation.

$\mathrm{b} P<.00001$. 
redirected or the treatment can be stopped. This process would also be true for interfraction adjustments. These contingencies would require that some form of tracking marker be placed on each ultrasound transducer during treatment sessions, which could easily be accomplished using standard optical or magnetic tracking techniques. ${ }^{40}$
It again should be noted that these markers will be on the patient's skin and will require no invasive procedures to introduce them.

Tracking the surface of the lung could be of interest if one wanted to use ultrasound to estimate the motion of a tumor that was within the lung parenchyma but did not

Fig. 4. A, Four-dimensional computed tomographic scan showing the exhale (or inhale) phase from 4-dimensional computed tomographic positioning with orthogonal transverse (left image) and sagittal (right image) positioning. The inhalation tumor volume is drawn and displayed as a red structure, and the exhalation position is displayed in yellow. The tumor motion was approximated to be $1.5 \mathrm{~cm}$ in both the anterior-posterior and cephalad-caudad direction. The total displacement is $21.2 \mathrm{~mm}$. B. Motion of the liver with the hepatocellular carcinoma measured by hand from the video images of the ultrasound study. The total displacement of the motion is represented by the yellow line. The position of the tumor in each image is marked with a white arrow. The left image is full inspiration, and the right image is full expiration. The length of the yellow line is $23 \mathrm{~mm}$. $\mathbf{C}$ (opposite page), Motion of the liver with the tumor from full inspiration (top left image) to full expiration (top right image). The images at the two extremes of the motion are shown with their corresponding positions on the time displacements marked with arrows. The region of the tumor is marked on the images with a red circle.

\section{A}
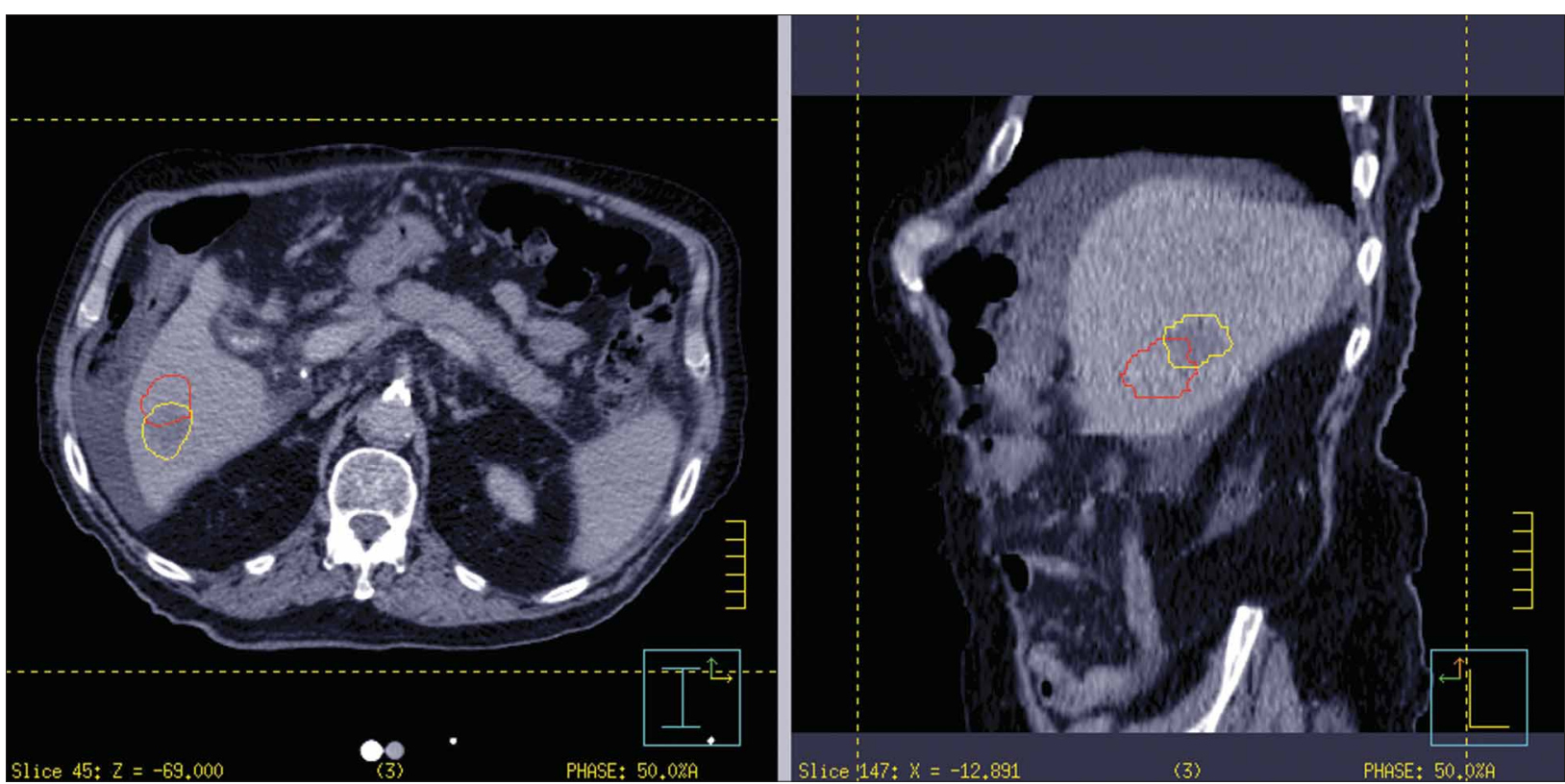

B
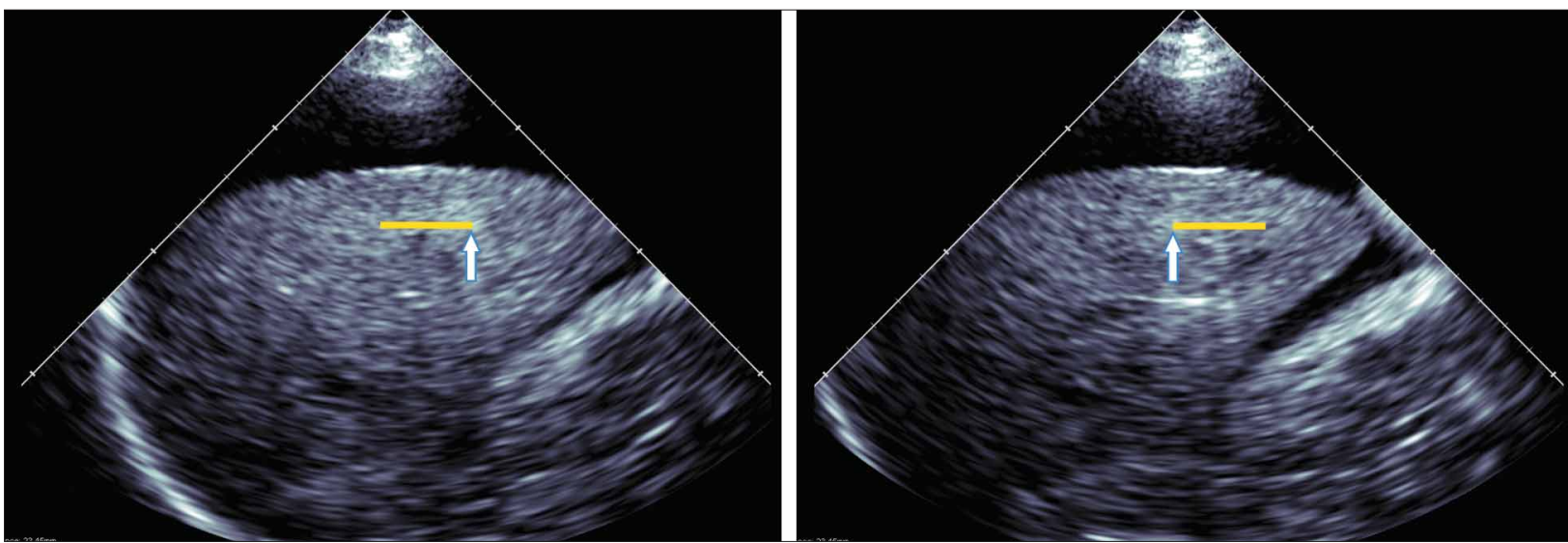
touch the surface of the lung, making it invisible to ultrasound. Sound does not enter air-filled lung, so the only part of the lung that is visible is the insonified surface. The surface of the lung is a large flat boundary compared to the wavelengths of ultrasound used (about 150-500 $\mu \mathrm{m}$ ). It produces a specular reflection, which is different from a standard speckle source composed of distributed scatterers. Specular reflections do not support speckle tracking along the reflector surface because there is no speckle variation. This lack of speckle as well as the substantial noise introduced at this boundary due to sound reverberations caused by the large air-soft tissue acoustic impedance difference makes tracking this surface challenging even though the lung boundary is moving with respiration.

Thus, without filtering, the received echoes are a combination of clutter signals, reflections from the large gassoft tissue surface, and signals from the moving lung surface. This combined signal can be difficult to track because of speckle decorrelation due to interference/noise such that the motion measurements can be biased toward the stronger gas-soft tissue component, which in this case is a large stationary signal. The clutter filter removes only the stationary components, leaving the signal corresponding to the motion of the lung surface.

\section{C}

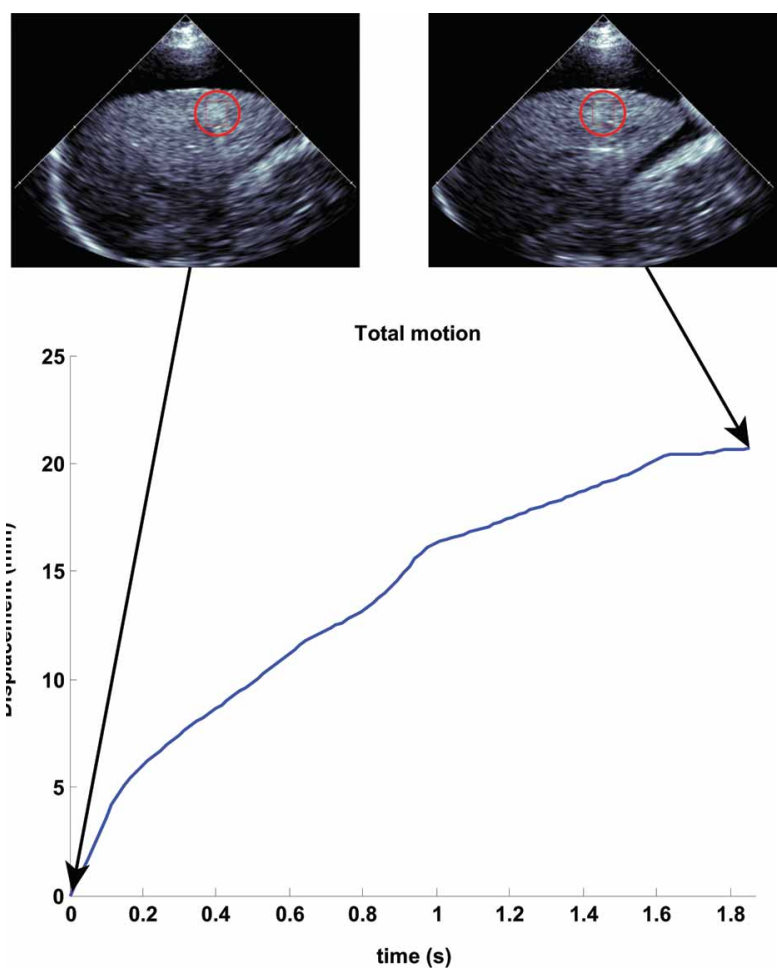

One challenge that has been identified with using speckle tracking to measure tissue displacements over time periods typical of radiation therapy is measurement drift due to biased tracking errors. ${ }^{28} \mathrm{~A}$ primary cause of these errors is small interframe motion, where the true tissue motion is less than the smallest resolved motion of the speckletracking algorithm, typically limited by noise or speckle coherence. Successive frames of small motion can result in considerable tracking drift. One solution is to adjust the acquisition frame rate to ensure trackable interframe motion. However, during radiation therapy monitoring and guidance, the interframe motion will change due to patient respiration and movement. In addition, adjusting the frame rate for the smallest expected motion may result in difficulties at larger tissue velocities due to speckle decorrelation or motion exceeding the tracking range of the speckle-tracking processing. Adaptive frame decimation offers an improved solution. ${ }^{41}$ Using this method, data are acquired at frame rates needed for fast tissue motion tracking, and the frame rate is adaptively reduced for slower motions based on speckle correlation. In addition, tracking of image regions that do not change shape or deform substantially over time (eg, tissue borders, blood vessels, etc) can be used to correct for drift in speckle-tracking estimates. ${ }^{28}$

At least 2 previous papers have used electronic $2 \mathrm{D}$ array probes for 3D speckle tracking, ${ }^{27,28}$ and 3D speckle tracking has been shown to be highly accurate. ${ }^{16}$ However, presently $2 \mathrm{D}$ ultrasound arrays are still largely developmental, extremely expensive, and not in general use. In this study, we performed our tracking in $2 \mathrm{D}$, and our results suggest that $2 \mathrm{D}$ tracking should easily suffice for the motions we would like to detect.

It is definitely possible that complex motion could cause speckle to decorrelate due to out-of-plane motion when using $2 \mathrm{D}$ tracking. Such occurrences would affect the accuracy and quality of motion tracking. However, correlation tracking is by nature a very good metric for assessing the accuracy of tracking, and correlation coefficient magnitudes are presently used to assess the quality of deformations during elasticity imaging. ${ }^{42,43}$ One could, in the same way, use the tracking correlation coefficient magnitudes to assess whether speckle tracking of organ motion during a radiation treatment session is reliable. If not, one could ignore the speckle-tracking motion estimates and revert to the previously used motion techniques such as 4DCT. Finally, given all of these considerations, organ tracking for radiation oncology monitoring could be initiated using $2 \mathrm{D}$ tracking with the conversion to $3 \mathrm{D}$ tracking occurring once $2 \mathrm{D}$ arrays are inexpensive and generally 
available. Whether $2 \mathrm{D}$ tracking is sufficient will require validation on an organ-by-organ basis.

Another obvious limitation of this study is that it was performed on only 2 healthy volunteers and 1 patient with a hepatocellular carcinoma. Although it is difficult to draw major conclusions on such small numbers, we believe that based on these preliminary results, ultrasound speckle tracking has the potential to monitor the motions of these organs, and such tracking is possible and feasible. Further studies will need to actually validate this technique, which could be a noninvasive way to track internal organ motion during treatment delivery to ensure tumor coverage and normal tissue sparing during radiotherapy.

These studies will have to overcome at least two difficulties we had in validating speckle tracking in this preliminary study, ie, our inability to validate the motion estimates of the lung surface and the inability to track true motion in the prostate. Even in the liver, where we could compare speckle tracking to the motion of true anatomic points during respiration, the number of cases is small. However, it should be possible to assess the robustness of speckle tracking in each of these circumstances in humans. In the liver, in addition to anatomic points, one can compare speckle tracking to interactive ultrasound-based tracking of $\mathrm{x}$-ray-visible implanted markers that are frequently placed to monitor respiratory motion during radiation treatment. These markers are visible on ultrasound imaging and would generate very well-defined points whose motions could be compared to speckle-tracking estimates. In the lung and particularly the prostate, externally placed magnetic beacons (Calypso Medical Technologies, Inc, Seattle, WA) can be inserted to monitor organ motions in $3 \mathrm{D}$ during radiation treatments. ${ }^{36}$ We can compare speckle-tracking motion estimates to those of these beacons as ground truth.

In conclusion, we have demonstrated the potential application of ultrasound speckle tracking to monitor motion of the liver, prostate, and lung surface as part of motion management strategies for radiotherapy. The method worked well in a patient with hepatocellular carcinoma. The method is robust, accurate, minimally user dependent, and totally safe. Further studies tracking tumor motions in more patients are planned.

\section{References}

1. Feng M, Balter JM, Normolle D, et al. Characterization of pancreatic tumor motion using cine MRI: surrogates for tumor position should be used with caution. Int J Radiat Oncol Biol Phys 2009; 74:884-891.
2. Weiss E, Wijesooriya K, Dill SV, Keall PJ. Tumor and normal tissue motion in the thorax during respiration: analysis of volumetric and positional variations using 4D CT. Int J Radiat Oncol Biol Phys 2007; 67:296-307.

3. Case RB, Moseley DJ, Sonke JJ, et al. Interfraction and intrafraction changes in amplitude of breathing motion in stereotactic liver radiotherapy. Int J Radiat Oncol Biol Phys 2010; 77:918-925.

4. Noel C, Parikh PJ, Roy M, et al. Prediction of intrafraction prostate motion: accuracy of pre- and post-treatment imaging and intermittent imaging. Int J Radiat Oncol Biol Phys 2009; 73:692-698.

5. Jin J-Y,Ajlouni M, Chen Q, Yin F-F, Movsas B. A technique of using gatedCT images to determine internal target volume (ITV) for fractionated stereotactic lung radiotherapy. Radiother Oncol 2006; 78:177-184.

6. Liu HH, Balter PA, Tutt T, et al. Assessing respiration-induced tumor motion and internal target volume using four-dimensional computed tomography for radiotherapy of lung cancer. Int J Radiat Oncol Biol Phys 2007; 68:531-540.

7. Vedam SS, Keall PJ, Kini VR, Mohan R. Determining parameters for respiration-gated radiotherapy. Med Phys 2001; 28:2139-2146.

8. Hunjan S, Starkschall G, Prado K, Dong L, Balter P. Lack of correlation between external fiducial positions and internal tumor positions during breath-hold CT. Int J Radiat Oncol Biol Phys 2010; 76:1586-1591.

9. Koch N, Liu HH, Starkschall G, et al. Evaluation of internal lung motion for respiratory-gated radiotherapy using MRI, part I: correlating internal lung motion with skin fiducial motion. Int J Radiat Oncol Biol Phys 2004; 60:1459-1472

10. Ozhasoglu C, Murphy MJ. Issues in respiratory motion compensation during external-beam radiotherapy. Int J Radiat Oncol Biol Phys 2002; 52:1389-1399.

11. Balter JM, Brock KK, Litzenberg DW, et al. Daily targeting of intrahepatic tumors for radiotherapy. Int J Radiat Oncol Biol Phys 2002; 52:266271.

12. Chang DT, Schellenberg D, Shen J, et al. Stereotactic radiotherapy for unresectable adenocarcinoma of the pancreas. Cancer 2009; 115:665-672.

13. Shirato H, Shimizu S, Kitamura K, et al. Four-dimensional treatment planning and fluoroscopic real-time tumor tracking radiotherapy for moving tumor. Int J Radiat Oncol Biol Phys 2000; 48:435-442.

14. Chen EJ,Adler RS, Carson PL, Jenkins WK, O’Brien WD Jr. Ultrasound tissue displacement imaging with application to breast cancer. Ultrasound Med Biol 1995; 21:1153-1162.

15. Chen J-F, Fowlkes JB, Carson PL, Rubin JM. Determination of scan-plane motion using speckle decorrelation: theoretical considerations and initial test. Int J Imaging Syst Technol 1997; 8:38-44.

16. Chen X, Xie H, Erkamp R, et al. 3-D correlation-based speckle tracking. Ultrason Imaging 2004; 27:21-36.

17. Lubinski MA, Emelianov SY, O’Donnell M. Speckle tracking methods for ultrasonic elasticity imaging using short time correlation. IEEE Trans Ultrason Ferroelectr Freq Control 1999; 46:82-96.

18. O'Donnell M, Emelianov SY, Skovoroda AR, Lubinski MA, WeitzelWF, Wiggins RC. Quantitative elasticity imaging. Proc IEEE Ultrason Symp 1993; 2:893-903. 
19. Ophir J, Cespedes I, Ponnekanti H, Yazdi Y, Li X. Elastography: a quantitative method for imaging the elasticity of biological tissues. Ultrason Imaging 1991; 13:111-134.

20. Konofagou E, Ophir J. A new elastographic method for estimation and imaging of lateral displacements, lateral strains, corrected axial strains and Poisson's ratios in tissues. Ultrasound Med Biol 1998; 24:1183-1199.

21. Maurice RL, Bertrand M. Lagrangian speckle model and tissue-motion estimation: theory. IEEE Trans Med Imaging 1999; 18:593-603.

22. Trahey GE, Allison JW, von Ramm OT. Angle-independent ultrasonic detection of blood flow. IEEE Trans Biomed Eng 1987; 34:965-967.

23. Wagner RF, Smith SW, Sandrik JM, Lopez H. Statistics of speckle in ultrasound B-scans. IEEE Trans Son Ultrason 1983; 30:156-163.

24. D’Hooge J, Heimdal A, Jamal F, et al. Regional strain and strain rate measurements by cardiac ultrasound: principles, implementation and limitations. Eur JEchocardiogr 2000; 1:154-170.

25. Weng L, Tirumalai AP, Lowery CM, et al. US extended-field-of-view imaging technology. Radiology 1997; 203:877-880.

26. Hsu A, Miller NR, Evans PM, Bamber JC, Webb S. Feasibility of using ultrasound for real-time tracking during radiotherapy. Med Phys 2005; 32:1500-1512.

27. Harris EJ, Miller NR, Bamber JC, Evans PM, Symonds-Tayler JR. Performance of ultrasound-based measurement of $3 \mathrm{D}$ displacement using a curvilinear probe for organ motion tracking. Phys Med Biol 2007; 52:5683-5703.

28. Harris EJ,Miller NR, Bamber JC, Symonds-TaylerJR, Evans PM. Speckle tracking in a phantom and feature-based tracking in liver in the presence of respiratory motion using 4D ultrasound. Phys Med Biol 2010; 55:33633380.

29. Jacso F, Kouznetsov A, Smith WL. Development and evaluation of an ultrasound-guided tracking and gating system for hepatic radiotherapy. Med Phys 2009; 36:5633-5640.

30. Ashraf M, Carlson M, Newey C, et al. Ultrasound speckle tracking system using radiofrequency data for simultaneous computation of strain and strain rate: validation against sonomicrometry [abstract]. Circulation 2010; 122(suppl):A9116.

31. Brock KK, Hollister SJ, Dawson LA, Balter JM. Technical note: creating a four-dimensional model of the liver using finite element analysis. Med Phys 2002; 29: 1403-1405.

32. Brock KK, McShan DL, Ten Haken RK, Hollister SJ, Dawson LA, Balter JM. Inclusion of organ deformation in dose calculations. Med Phys 2003; 30:290-295.

33. Brock KM, Balter JM, Dawson LA, Kessler ML, Meyer CR. Automated generation of a four-dimensional model of the liver using warping and mutual information. Med Phys 2003; 30:1128-1133.

34. Rohlfing T, Maurer CRJr, O’Dell WG, Zhong J. Modeling liver motion and deformation during the respiratory cycle using intensity-based nonrigid registration of gated MR images. Med Phys 2004; 31:427-432.

35. Balter JM, Sandler HM, Lam K, Bree RL, Lichter AS, Ten Haken RK. Measurement of prostate movement over the course of routine radiotherapy using implanted markers. Int J Radiat Oncol Biol Phys 1995; $31: 113-118$
36. Langen KM, Willoughby TR, Meeks SL, et al. Observations on real-time prostate gland motion using electromagnetic tracking. Int J Radiat Oncol Biol Phys 2008; 71:1084-1090.

37. Kroger K, Massalha K, Dobonici G, Rudofsky G. SieScape: a new sonographic dimension with fictive images. Ultrasound Med Biol 1998; 24:1125-1129.

38. Trahey GE, Smith SW, von Ramm OT. Speckle pattern correlation with lateral aperture translation: experimental results and implications for spatial compounding. IEEE Trans Ultrason Ferroelectr Freq Control 1986; 33:257-264.

39. Tokuda J, Schmitt M, Sun Y, et al. Lung motion and volume measurement by dynamic 3D MRI using a 128-channel receiver coil. Acad Radiol 2009; 16:22-27.

40. Dieterich S, TangJ, Rodgers J, Cleary K. Skin respiratory motion tracking for stereotactic radiosurgery using the CyberKnife. Int Congress Ser 2003; 1256:130-136.

41. Lubinski MA, Emelianov SY, O’Donnell M. Adaptive strain estimation using retrospective processing. IEEE Trans Ultrason Ferroelectr Freq Control 1999; 46:97-107.

42. Booi RC, Carson PL, O’Donnell M, Richards MS, Rubin JM. Diagnosing cysts with correlation coefficient images from 2-dimensional freehand elastography.J Ultrasound Med 2007; 26:1201-1207.

43. Booi RC, Carson PL, O’Donnell M, Roubidoux MA, Hall AL, Rubin JM. Characterization of cysts using differential correlation coefficient values from two-dimensional breast elastography: preliminary study. Ultrasound Med Biol 2008; 34:12-21. 